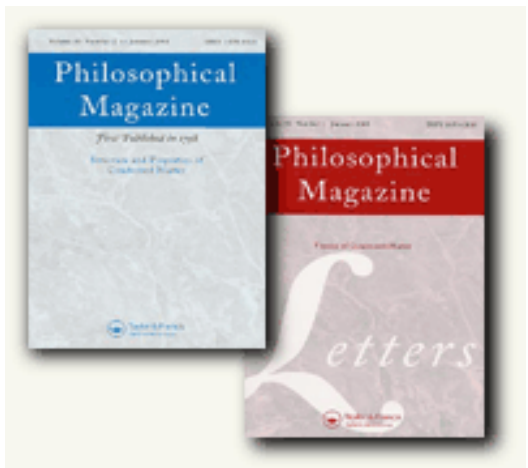

\title{
A multi-scale model of domain wall velocities based on ab initio parameters
}

\begin{tabular}{|r|l|}
\hline Journal: & Philosophical Magazine \& Philosophical Magazine Letters \\
\hline Manuscript ID: & TPHM-10-Sep-0419 \\
\hline Journal Selection: & Philosophical Magazine \\
\hline Author: & $17-$ Sep-2010 \\
\hline Complete List of Authors: & $\begin{array}{l}\text { Weinberger, Peter; Center for Computational Nanoscience } \\
\text { Vedmedenko, Elena; Institut für Angewandte Physik, Universität } \\
\text { Hamburg } \\
\text { Wieser, Robert; Institut für Angewandte Physik, Universität } \\
\text { Hamburg } \\
\text { Wiesendanger, Roland; Institut für Angewandte Physik, Universität } \\
\text { Hamburg }\end{array}$ \\
\hline Keywords: & $\begin{array}{l}\text { magnetism, magnetization, magnetization dynamics, domain } \\
\text { structure }\end{array}$ \\
\hline Keywords (user supplied): & \begin{tabular}{l} 
domain wall velocities \\
\hline
\end{tabular} \\
\hline
\end{tabular}

\section{SCHOLARONE \\ Manuscripts}




\title{
A multi-scale model of domain wall velocities based on ab initio parameters
}

\author{
P. Weinberger, ${ }^{1}$ E. Y. Vedmedenko, ${ }^{2}$ R. Wieser,${ }^{2}$ and R. Wiesendanger ${ }^{2}$ \\ ${ }^{1}$ Center for Computational Nanoscience, Seilerstätte 10/22, A1010 Vienna, Austria \\ ${ }^{2}$ Institut für Angewandte Physik, Universität Hamburg, Jungiusstraße 11, 20355 Hamburg, Germany
}

\begin{abstract}
A new approach is presented to evaluate the velocity of field-driven domain walls by means of ab-initio parameters. This approach makes intensive use of multi-scaling by means of (a) mapping of domain wall formation energies obtained in terms of a fully relativistic method onto a LandauGinzburg-type expression, and (b) applying the Landau-Lifshitz-Gilbert equation to evaluate the time needed to move domain walls. In comparison with the "classical" expression for the domain wall velocity originally proposed by Landau and Lifshitz, according to which the velocity increases with increasing width of the domain wall, three different types of magnetic alloys, namely permalloy $\left(\mathrm{Ni}_{85} \mathrm{Fe}_{15}\right), \mathrm{Co}_{x} \mathrm{Ni}_{1-x}$ and $\mathrm{Co}_{x} \mathrm{Pd}_{1-x}$ are analyzed. It is shown that the Landau-Lifshitz expression for the velocity seems to be valid whenever the slopes of the exchange and the anisotropy energy with respect to the concentration are either both increasing or both decreasing.
\end{abstract}

\section{INTRODUCTION}

Current-driven domain wall (DW) motions seem to raise a lot of interest because of their possible use in completely new types of storage media [1-7]. For their theoretical description and modelling presently almost exclusively only micromagnetic approaches [5, 7-9] are applied, which usually rely on a (macroscopic) Landau-Lifshitz-Gilbert (LLG) equation with an additional phenomenological spin-torque term $[7,9]$ that contains a so-called non-adiabaticity parameter $\beta$, supposed to describe the alignment of the spins of the charge carriers with those of the wall within a classical view. Since - like all other quantities in micromagnetic schemes - $\beta$ inherently is introduced parametrically, a materials-specific description of DW motions seems to be quite likely out of reach, even if all parameters are "borrowed" from experiment. Interestingly enough, it turned out that while according to the "classical" Landau-Lifshitz expression [21] - [23] for the velocity of field-driven DW of length $L, v(L)$, increases with $L$, a micromagnetic simulation of $v(L)$ for current-driven DW's predicted independence of $L[8]$.

Although presently available $a b$ initio spin dynamics [10-12] and new multi-scale approaches [13-20] suggest that also quantum mechanical schemes are within reach, the difficulty to be encountered is that non-collinear magnetic structures require to include (at least) spin-orbit effects, which for domain walls because of their size is no easy task. Furthermore, since quantum mechanically well-defined spin torques and spin currents [24] are not likely to be available computationally in the near future on a level to be used for DW's, in the meantime a multi-scale approach is proposed for the description of DW motions in which the free energy is first evaluated fully relativistically on an ab-initio level and then - in the spirit of spin dynamics [10-12] - mapped onto the internal field in the LLG equation.

Several applications will demonstrate that indeed materials-specific DW velocities can be evaluated without assuming parameters beyond those inherent to density functional theory, i.e., beyond a standard parametrization of the density functional. A comparison with the Landau-Lifshitz expression for the velocity [21-23] will give further insight into the use of the suggested multi-scale approach.

\section{THEORETICAL DESCRIPTION}

Formation energy of domain walls

Suppose that $C_{0}$ and $C_{d}$ denote the following magnetic configurations in a system with only two-dimensional translational symmetry ("layered system"),

$$
\begin{aligned}
& C_{0}(L)=\left\{\vec{n}_{l}=\vec{z}, \quad \vec{n}_{k}=\vec{z}, \quad \vec{n}_{r}=\vec{z}, \quad k=1, L\right\}, \\
& C_{d}(L)=\left\{\vec{n}_{l}=\vec{z}, \quad \vec{n}_{k}, \quad \vec{n}_{r}=-\vec{z}, \quad k=1, L\right\},
\end{aligned}
$$

where $\vec{n}_{l}$ and $\vec{n}_{r}$ refer to the orientations of the magnetization in the "left" and the "right" domain, the $\vec{n}_{k}$ those in the atomic planes forming the wall, and $\vec{z}$ is parallel to the surface normal. In $C_{d}$ the orientation of the magnetization in the individual atomic planes changes continuously from $\vec{z}$ to $-\vec{z}$,

$$
\vec{n}_{k}=D\left(\Theta_{k}\right) \vec{z} \quad, \quad \vec{n}_{k}=D\left(\Theta_{k}\right) \vec{z} \quad, \quad \Phi_{k}=\pi k / L,
$$


$D\left(\Theta_{k}\right)$ being a rotation by an angle $\Theta_{k}$ around the in-plane $\vec{x}$-axis. It should be noted that for matters of simplicity in Eqs. (2) and (3) a simple sinusoidal wall profile is assumed. Clearly enough the use of any other, perhaps more realistic set $\left\{D\left(\Theta_{k}\right)\right\}$ is straightforward. For a given value of $L$ the domain wall formation energy is then given by

$$
E(L)=E\left(\mathcal{C}_{d}(L)\right)-E\left(\mathcal{C}_{0}(L)\right),
$$

where, when adopting the magnetic force theorem, $E\left(\mathcal{C}_{d}(L)\right)$ and $E\left(\mathcal{C}_{0}(L)\right)$ refer to grand-canonical potentials at $\mathrm{T}$ $=0$. If $c_{\alpha}^{p}$ denotes the respective concentrations of constituents $\mathcal{A}$ and $\mathcal{B}$ in layer $p$ of a substitutionally statistically disordered alloy, then in terms of the (inhomogeneous) Coherent Potential Approximation (CPA) for layered systems $[25,26] E(L)$ is given by

$$
\begin{gathered}
E(L)=\sum_{p=1}^{L} E^{p}(L)=\sum_{p=1}^{L} \sum_{\alpha=\mathcal{A}, \mathcal{B}} c_{\alpha}^{p}\left(E_{\alpha}^{p}\left(\mathcal{C}_{d}(L)\right)-E_{\alpha}^{p}\left(\mathcal{C}_{o}(L)\right)\right) \\
E_{\alpha}^{p}\left(\mathcal{C}_{i}(L)\right)=\int_{\epsilon_{b}}^{\epsilon_{F}} n_{\alpha}^{p}\left(\epsilon ; \mathcal{C}_{i}(L)\right)\left(\epsilon-\epsilon_{F}\right) d \epsilon
\end{gathered}
$$

where the $n_{\alpha}^{p}\left(\epsilon ; \mathcal{C}_{i}(L)\right)$ are the components of the layerwise projected DOS corresponding to the magnetic configuration $\mathcal{C}_{i}(L), \epsilon_{b}$ denotes the bottom of the valence band, and $\epsilon_{F}$ is the Fermi energy.

Phenomenologically, by using a Ginzburg-Landau-type expansion, the domain wall formation energy $\mathcal{E}(L)$ is defined by $[13,17]$

$$
\mathcal{E}(L)=A_{0}\left(\frac{A}{L}+K L\right)
$$

where $A_{0}$ is the area of the two-dimensional unit cell, and $A$ and $K$ are proportional to the exchange and magnetic anisotropy energy, respectively. From the condition $d \mathcal{E}(L) / d L=0$ it follows immediately that the equilibrium domain wall width $L_{0}$ is given by $L_{0}=\sqrt{A / K}$. The coefficients $A$ and $B$ in Eq. (7) can easily be obtained by evaluating free energies $E(L)$, see Eq. (5), at (at least) two different values of $L$. It should be noted that by using Eq. (7) a first multi-scale step is taken because a quantum mechanically defined quantity, namely the domain wall formation energy, is mapped without additional parameters on a phenomenological (macroscopic) quantity.

\section{Domain wall motions}

Clearly enough in the definition of magnetic configurations, see, e.g., Eq. (2), a domain wall is described "fixed" in coordinate space by an arbitrary choice of an origin in order to specify layer indices

$$
\begin{gathered}
C_{d}(L)=\left\{\left\{\vec{n}_{l}=\vec{z}\right\},\left\{D\left(\Theta_{1}\right) \vec{z}, D\left(\Theta_{2}\right) \vec{z}, \ldots,\right.\right. \\
\left.\left.D\left(\Theta_{L}\right) \vec{z}\right\},\left\{\vec{n}_{r}=-\vec{z}\right\}\right\}, \\
l=0,-1,-2, \ldots-\infty, \quad r=L+1, L+2, \ldots, \infty .
\end{gathered}
$$

To shift such a domain wall by one ML, i.e., to "make it move", one has to switch from $C_{d}(L)$ to $C_{d}(L)$

$$
\begin{gathered}
C_{d}(L)=\left\{\left\{\vec{n}_{l}=\vec{z}\right\},\left\{\vec{z}, D\left(\Theta_{2}\right) \vec{z}, \ldots, D\left(\Theta_{L}\right) \vec{z},\right.\right. \\
\left.\left.D\left(\Theta_{L+1}\right) \vec{z}, D\left(\Theta_{L+1}\right) \vec{z}\right\},\left\{\vec{n}_{r}=-\vec{z}\right\}\right\}, \\
l=1,0,-1,-2, \ldots-\infty, \quad r=L+2, L+3, \ldots, \infty,
\end{gathered}
$$

After the "move", i.e., being again in "equilibrium", of course the following condition has to apply,

$$
E\left(\mathcal{C}_{d}(L)\right) \equiv E\left(\mathcal{C}_{d}^{\prime}(L)\right)
$$

which in turn implies that a simple re-indexing of layers will not describe a domain wall motion.

Consider now a Taylor series expansion,

$$
f(u)=\sum_{n=1}^{P} \frac{f^{(n)}\left(u_{0}\right)}{n !}\left(u-u_{0}\right)^{n} \quad, \quad f^{(n)}=\frac{d^{(n)}}{d u^{n}},
$$


for the layer-resolved band energies, see Eqs. (5) and (6), reformulated in Eq. (12) such that the layer-index is cast into multiples of the interlayer distance $d_{\perp}$

$$
f:\left\{x_{k}=k d_{\perp}\right\} \rightarrow\left\{E^{k}(L)\right\} \equiv\left\{E\left(L ; x_{k}\right)\right\} .
$$

Now it is easy to see that in analogy to the approach suggested in Ref. [27] in terms of Eq. (11) layer-resolved band energies shifted along the surface normal can be written as

$$
E\left(L ; u_{k}\right)=E\left(L ; x_{k}\right)+a_{k}(L)\left(u_{k}-x_{k}\right)+b_{k}(L)\left(u_{k}-x_{k}\right)^{2}+c_{k}(L)\left(u_{k}-x_{k}\right)^{3} \ldots, \quad k=1, L .
$$

In order to move a domain wall by one monolayer $u_{k}$ has to fulfill the condition

$$
\begin{aligned}
u_{k}-x_{k} & =d_{\perp} \cos (\Delta \Theta), \\
\Delta \Theta & =\Theta_{k \pm 1}-\Theta_{k} .
\end{aligned}
$$

It should be noted that $u_{k}$ corresponds to the $z$-like component of a displacement operator in coordinate space of the type $\vec{u}_{k}=\left(0,0, u_{k}\right)$. In fact in a "parent" (simple) lattice $d_{\perp}$ refers to a translation, which in turn is followed by a rotation of the magnetization with respect to the difference angle between the orientation of the magnetization in layers $k$ and $k \pm 1$, for details, see Ref. [25]. The error $W(L)$ of this numerical procedure can easily be checked by evaluating the difference between the band energy before and after the shift

$$
W(L)=\left|E\left(\mathcal{C}_{d}^{\prime}(L)\right)-E\left(\mathcal{C}_{d}(L)\right)\right| / E\left(\mathcal{C}_{d}(L)\right)
$$

since according to Eq. (10) $W(L)$ should be zero. Quite clearly the above outlined procedure can in principle be applied as often as necessary, i.e., a domain wall can easily be moved over a distance $p d_{\perp}$ along the surface normal. It is a scheme that frequently is used as "inbetween step" in molecular dynamics as a scaling procedure.

\section{Landau-Lifshitz-Gilbert equation}

As already mentioned in the introduction, in order to estimate the time needed to move a domain wall of given width $L$ at present only the below (layer-resolved) equation of motion is available

$$
\frac{d \vec{m}_{i}(L, t)}{d t}=-\gamma \vec{m}_{i}(L, t) \times \vec{H}^{\mathrm{eff}}\left(L, \vec{u}_{i}\right)+\alpha \vec{m}_{i}(L, t) \times\left(\vec{m}_{i}(L, t) \times \vec{H}^{\mathrm{eff}}\left(L, \vec{u}_{i}\right)\right)+\vec{\lambda}_{i}(L), \quad i=1, L,
$$

where the case $\vec{\lambda}_{i}(L)=0$ refers to the "classical" LLG equation, successfully used in ab-initio spin dynamics [10-12], while

$$
\vec{\lambda}_{i}(L)=\frac{1+\beta \alpha}{\left(1+\alpha^{2}\right)} j_{z}(L) \vec{m}_{i}(L, t) \times\left(\vec{m}_{i}(L, t) \times \frac{\partial \vec{m}_{i}(L, t)}{\partial z}\right)-\frac{\alpha-\beta}{\left(1+\alpha^{2}\right)} j_{z}(L) \vec{m}_{i}(L, t) \times \frac{\partial \vec{m}_{i}(L, t)}{\partial z}
$$

corresponds to a phenomenologically augmented LLG equation, which usually is termed "generalized" LLG equation. In Eq. (17) $\alpha$ is the so-called Gilbert damping factor and $\gamma$ the gyromagnetic ratio; in Eq. (18) the current density $j_{z}(L)$ is given by $\left(P(L) g \mu_{B} /\left(2 e M_{s}\right)\right) \vec{j}(L)$ with $\vec{j}(L)$ being the current density, $P(L)$ the (non-relativistic) "spin" polarization, $M_{s}$ the saturation magnetization, and $\beta$ corresponds to the already mentioned non-adiabaticity parameter. [7]

Since for the time being only $\vec{H}_{i}^{\text {eff }}(L, \vec{u})$,

$$
\begin{gathered}
\vec{H}_{i}^{\mathrm{eff}}(L, \vec{u})=-\frac{\partial\left(\Delta E^{i}(L, u)\right)}{\partial \vec{u}}, \\
H_{i, x}^{\mathrm{eff}}\left(L, u_{x}\right)=H_{i, y}^{\mathrm{eff}}\left(L, u_{y}\right)=0 \quad, \quad \forall u, \\
H_{i, z}^{\mathrm{eff}}(L, u)=-\frac{d\left(\Delta E^{i}(L, u)\right)}{d u},
\end{gathered}
$$

where

$$
\Delta E^{i}(L, u)=E^{i}(L, u)-E^{i}\left(L, u_{0}\right)
$$


FIG. 1: (colour online) $\mathrm{Ni}_{85} \mathrm{Fe}_{15}$. Top: Original (unshifted) layer-resolved band energies for $L_{0}=1038 \mathrm{ML}$. The middle and the bottom entries refer to the band energies corresponding to the "left" and "right" edge of the original domain wall (full line) and the one shifted by 1 ML (dashed line).

can be associated with quantum mechanically well-defined quantities [28], in the following exclusively the "classical" LLG equation is applied, i.e., in Eq. (17) $\vec{\lambda}_{i}=0, \forall i$. Furthermore, in order to evaluate the time needed to change the orientation of the magnetization by $\Delta \Theta$ usually $[10-12,24]$ only the relaxation term in the LLG equation needs to be considered,

$$
\begin{aligned}
& m_{0} \frac{d n_{x}^{i}(L, t)}{d t}=0 \\
& m_{0} \frac{d n_{y}^{i}(L, t)}{d t}=-\alpha n_{y}^{i}(L, t) n_{z}^{i}(L, t) H_{i, z}^{\mathrm{eff}}(L, u) \\
& m_{0} \frac{d n_{\mathrm{z}}(L, t)}{d t}=+\alpha\left(n_{y}^{i}(L, t)\right)^{2} H_{i, z}^{\mathrm{eff}}(L, u)
\end{aligned}
$$

where

$$
\vec{n}^{i}(L, t)=\vec{m}^{i}(L, t) / m_{0} \quad, \quad m_{0}=\sum_{\alpha=A, B} c_{\alpha}^{i} m_{\alpha}^{i} \quad, \quad \forall L, t
$$

In Eq. (26) the $m_{\alpha}^{i}$ are the layer- and component-resolved magnetic moments and the $c_{\alpha}^{i}$ the corresponding concentrations of species $\mathcal{A}$ and $\mathcal{B}$. Since the magnitude of the magnetic moments is supposed to remain constant,

$$
\left(n_{y}^{i}(L, t)\right)^{2}+\left(n_{z}^{i}(L, t)\right)^{2}=1
$$



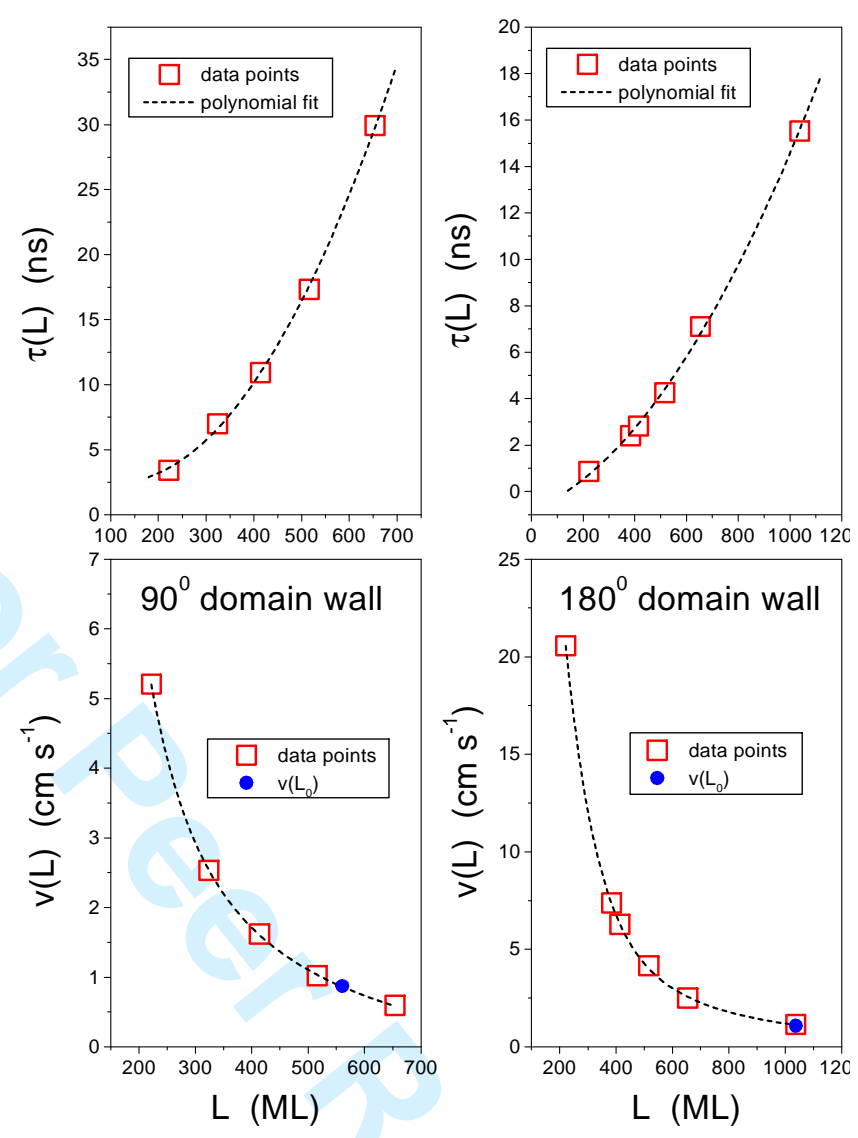

FIG. 2: (colour oneline) $\mathrm{Ni}_{85} \mathrm{Fe}_{15}$. Top row: fit of $\tau(L)$ by a second order polynomial. Bottom row: domain wall velocities. Displayed are the cases of a $90^{\circ}$ (left column) and a $180^{\circ}$ (right column) domain wall.

Eq. (25) reduces to

$$
\frac{d n_{z}^{i}(L, t)}{d t}=\left(1-\left(n_{z}^{i}(L, t)\right)^{2}\right) \times\left[\frac{\alpha}{m_{0}} H_{i, z}^{\mathrm{eff}}(L, u)\right] .
$$

By using the first 4 expansion coefficients in Eq. (13) the integration of Eq. (27) can now be performed using exactly the approach discussed in Ref. [29].

In the case of the "generalized" LLG equation, see Eq. (18), $\lambda_{i, z}$ would be of the form

$$
\lambda_{i, z}=\frac{1+\beta(L) \alpha}{\left(1+\alpha^{2}\right)} j_{z}(L) \frac{\partial n_{z}^{i}(L, t)}{\partial z},
$$

from which one immediately can see that $H_{i, z}^{e f f}$ and $\partial n_{z}^{i}(L, t) / \partial z$ are of opposite sign with $\alpha$ appearing in both terms of Eq. (27). It is important to note that by using Eq. (27) its implicitly assumed that during the motion of a domain wall its profile does not change. This restriction, however, can only be overcome by actually calculating directly for a given length $L$ the $n_{z}^{i}(L, t)$ as a function of $t$ selfconsistently within Density Functional Theory, a procedure that up to now is only possible for small clusters of magnetic atoms on metallic surfaces, see, e.g., [11]. 


\section{Velocity}

The velocity $v(L)$ of the motion of a domain wall of width $L$ can now easily be calculated, simply by making use of the relation

$$
v(L)=\frac{d_{\perp}}{\tau(L)},
$$

where $\tau(L)$ is the (minimal) time needed to move a domain wall of width $L$ over the distance of one monolayer,

$$
\tau(L)=\max \left\{\tau_{i}(L)\right\}
$$

and $d_{\perp}$ is the interlayer spacing (constant). In order to obtain $L_{0}$ in principle the domain wall formation energy $E(L)$ is needed only at two different $L$. This suggests to fit $\tau(L)$ by a second order polynomial of the form $y=a x+b x^{2}$ in order to obtain $\tau\left(L_{0}\right)$. Furthermore, since the changes in the layer-resolved band energies are biggest at the very beginning (or end) of a domain wall, it turns out that when moving a domain wall to the right, see Eq. $(14), \tau\left(L_{0}\right)$ is determined by $\tau_{1}\left(L_{0}\right)$, where $\tau_{1}\left(L_{0}\right)$ refers to the very first atomic layer in the domain wall, namely to the largest layer-dependent shift time.

\section{MULTI-SCALE PROCEDURES}

Before going ahead with applications it seems to be useful to summarize all multi-scale procedures interduced up-to-now, see Table I.

\begin{tabular}{|l|l|l|l|l|l|}
\hline step & microscopic & & macroscopic & seeked & procedure \\
\hline \hline 1 & Eq. 5: $E(L)$ & $\Longrightarrow$ & Eq. 7: $E(L)$ & $L_{0}$ & "Ginzburg-Landau" \\
\hline 2 & Eq. $22: \Delta E^{i}(L, u)$ & $\Longrightarrow$ & Eq. 19: $\vec{H}_{i}^{\text {eff }}(L, \vec{u})$ & $\tau_{i}(L)$ & "Landau-Lifshitz-Gilbert" \\
\hline & & & & $\Downarrow$ & \\
\hline & & & & $\tau\left(L_{0}\right)$ & computational fit \\
\hline
\end{tabular}

TABLE I: Summary of all multi-scale procedures and computational fits applied

\section{APPLICATIONS}

In this section three different systems are considered in order to illustrate the above approach and to investigate its relation to the "classical" Landau-Lifshitz expression [21] - [23] for the velocity,

$$
v=\frac{\gamma}{\alpha} M_{s} \sqrt{\frac{A}{K}}=\frac{\gamma}{\alpha} M_{s} L_{0}
$$

in which any spin-torque action is neglected. Since in Eq. (30) $v$ is proportional to the equilibrium width $L_{0}$ this implies that thicker domain walls move faster than thinner ones.

In the following, the velocities of 90 and 180 degree domain walls [19] in permalloy $\mathrm{Ni}_{85} \mathrm{Fe}_{15}(100)$ are investigated, and then two different magnetic substitutional alloys, namely $\mathrm{Co}_{x} \mathrm{Ni}_{1-x}(100)$ [18] and $\mathrm{Co}_{x} \mathrm{Pd}_{1-x}(111)$, [20] by varying the concentration of Co.

\section{Permalloy $\left(\mathrm{Ni}_{85} \mathrm{Fe}_{15}\right)$}

Fig. 1 illustrates the case of moving a $180^{\circ}$ domain wall of equilibrium width $L_{0}$ in $\mathrm{Ni}_{85} \mathrm{Fe}_{15}$, see Eq. (8), one ML to the right. It is interesting to note (top of this figure showing the original domain wall in terms of all layer-resolved band energies) that there is a rapid change in layer-resolved band energies only at the very beginning and the very end of the domain wall covering a region of about $10 \AA$ thickness. Furthermore, an in-plane anisotropy can be seen in terms 
of the maximum in the middle of the domain wall exactly when the direction of the magnetization is perpendicular to $\vec{z}$.

By using a Taylor expansion of order 5 a rather accurate shift by one ML is achieved, see the lower parts of Fig. 1, in which only the very beginning (middle entry) and the very end (bottom entry) of the original and the shifted domain wall are displayed. The lower parts of Fig. 1 prove that the domain wall was indeed shifted by $d_{\perp}$ to the right. Considering that the individual layer-resolved band energies $E^{i}$ are already of the order of $\mu \mathrm{eV}$ the remaining inaccuracies can hardly be removed, since both the layer-resolved energies [25, 26] as well as the derivatives in Eq. (11) result from numerical procedures. Despite these inherent inaccuracies it turns out that the error defined in Eq. (16) amounts to only $0.0009 \%$.

In Fig. 2 the mentioned polynomial fit of $\tau(L)$ is illustrated together with the resulting domain wall velocities $v(L)$. As can be seen this polynomial fit is indeed quite accurate. It seems therefore not necessary to evaluate the domain wall energy exactly at the equilibrium width as shown in Fig. 2 for the case of a $180^{\circ}$ domain wall in $\mathrm{Ni}_{85} \mathrm{Fe}_{15}$.

In Table II a detailed analysis for the two types of domain walls in permalloy is given. From this table it can be seen that essentially only the exchange energy $A$ is changing when going from a $90^{\circ}$ domain wall to a $180^{\circ}$ one $[19]$.

\begin{tabular}{|l|l|l|l|}
\hline & unit & $90^{\circ}$ & $180^{\circ}$ \\
\hline$A$ & $\mathrm{meV}$ & 2.3030 & 9.1514 \\
\hline$B$ & $\mu \mathrm{eV}$ & $0.72426 .10^{-2}$ & $0.71895 .10^{-2}$ \\
\hline$L_{0}$ & $\mathrm{ML}$ & 564 & 1038 \\
\hline$\tau\left(L_{0}\right)$ & $\mathrm{ns}$ & 20.28 & 16.43 \\
\hline$v\left(L_{0}\right)$ & $\mathrm{cm} \mathrm{s}^{-1}$ & 0.87 & 1.08 \\
\hline
\end{tabular}

TABLE II: Comparison between a $90^{\circ}$ and a $180^{\circ}$ domain wall in permalloy. The values for $\tau\left(L_{0}\right)$ and $v\left(L_{0}\right)$ correspond to a Gilbert damping factor of 1 .

From Table II it follows that the ratio $w$ between the two velocities is 1.23, while in terms of Eq. $(30), w \simeq$ $L_{0}(180) / L_{0}(90)$, this ratio amounts to about 2. Quite obviously for permalloy (at least for $\mathrm{Ni}_{85} \mathrm{Fe}_{15}$ ) this implies that as predicted by the Landau-Lifshitz relation thicker domain walls move indeed faster than thinner ones.

\section{$\mathrm{Co}_{x} \mathrm{Ni}_{1-x}$}

In $\mathrm{Co}_{x} \mathrm{Ni}_{1-x}$ there seem to be two different regimes of changes in $K(x)$ with respect to the concentration [19], see the insets in Fig. 3. As long as the sign of the slope of $A(x)$ and $K(x)$ with respect to $x$ is the same, i.e., when $0.4<x \leq 0.8$, a behavior as predicted by Eq. (30) is found. As is well-known for $x>0.8$ structural changes start to set in ( $\mathrm{Co}$ is hcp, while $\mathrm{Co}_{x} \mathrm{Ni}_{1-x}$ is fcc), so it seems that all considerations using a parent fcc lattice should be confined to about $x \leq 0.8$. In the range of $0 \leq x \leq 0.3$, however, $K(x)$ increases rapidly with decreasing $x$ while $A(x)$ decreases, see Fig. 5. In this regime the "classical description" $v\left(x, L_{0}\right) \sim L_{0}(x)$ in Eq. (30) seems to be of no great help.

$$
\mathrm{Co}_{x} \mathbf{P d}_{1-x}
$$

In $\mathrm{Co}_{x} \mathrm{Pd}_{1-x}$ there are quite a few concentration regimes in which $K(x)$ changes rapidly [20], see the left column in Fig. 5. Only for small values of $x$ the slopes of the concentration-dependent constants $A(x)$ and $K(x)$ have the same sign. This then is the (only) regime in which the velocity increases with the domain wall width, see Fig. 5 . For all other concentrations of Co, $v\left(x, L_{0}\right)$ is not proportional to $L_{0}(x)$, i.e., is not of Landau-Lifshitz type.

\section{CONCLUSION}

In the present paper an approach is introduced to evaluate by means of multi-scale schemes the velocity of domain wall motions in terms of ab-initio derived parameters. The phenomenological parts of the scheme are based on (a) the evaluation of equilibrium domain wall thicknesses $L_{0}(x)$ in terms of a Ginzburg-Landau-type expansion, see Eq. (7),[13] and (b) the Landau-Lifshitz-Gilbert equation to obtain shift times, see Eq. (27) [10-12], [29]. Furthermore, a second order polynomial expansion is suggested in order to extrapolate these times to $L_{0}(x)$., see also Table I. The 


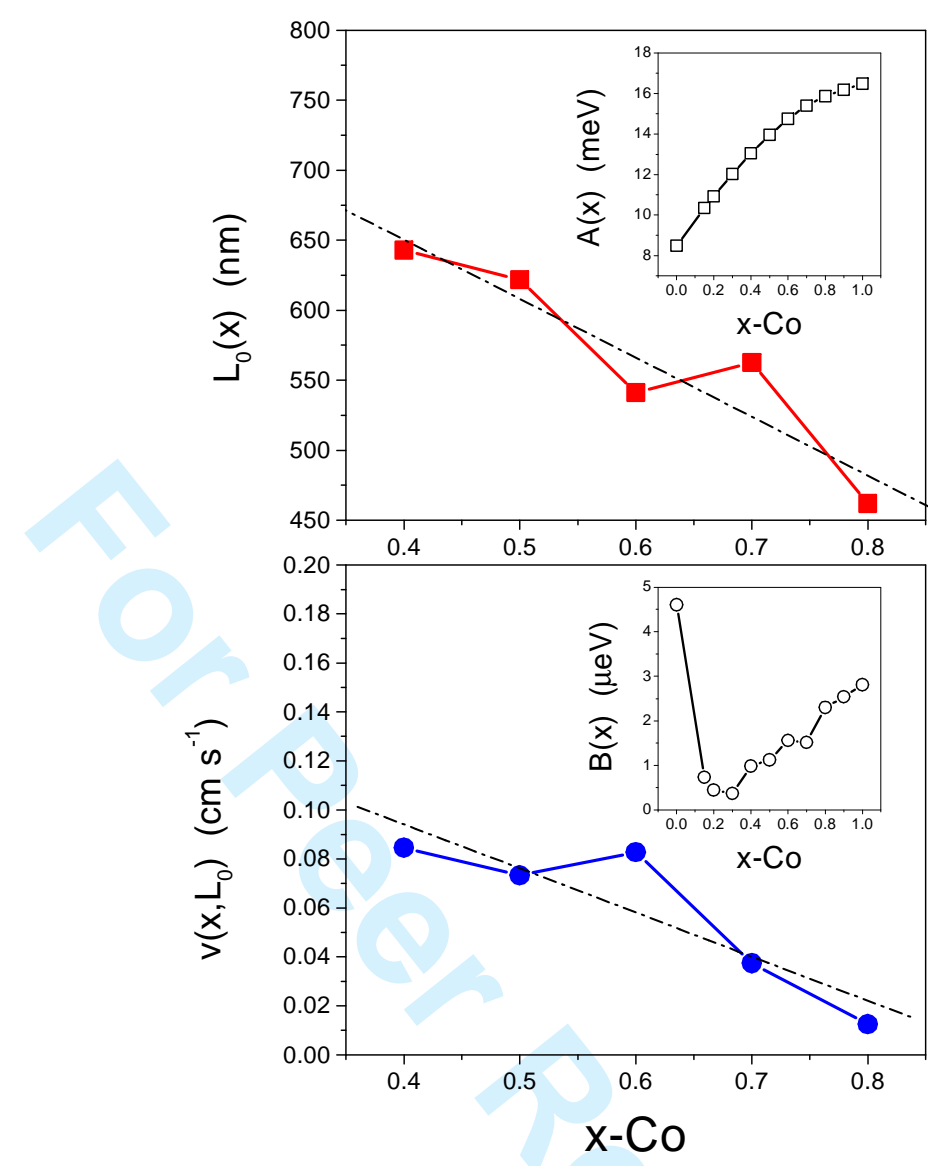

FIG. 3: (colour online) $\mathrm{Co}_{x} \mathrm{Ni}_{1-x}$. Equilibrium width $L_{0}(x)$ (top) and velocity $v\left(x, L_{0}\right)$ as indicated in the range of $0.4<$ $x_{C o} \leq 0.8$. The insets show the exchange (top) and anisotropy energy (top) data from Ref. [19].

microscopic parts consist of an evaluation of domain wall formation energies and their layer-wise contributions using a fully relativistic ab-initio approach, i.e., using the Dirac equation. The assumptions made were (a) that a domain wall profile was chosen which reproduces correctly the minimal domain wall energy, (b) that this profile does not change during the motion of domain wall, and (c) a particuar value for the Gilbert damping term, $\alpha$, has to be picked. The first assumption can easily be checked by varying the profile and evaluating the domain wall energy at $L_{0}$. The second assumption can presently not be verified, since in principle also the influence of the applied external field that causes a domain wall to move has to be taken into account [24]. Clearly also in micromagnetic schemes a value for $\alpha$ has to be chosen.

For permalloy a kind of Landau-Lifshitz behavior, see Eq. (30), is found, which is only partially the case in $\mathrm{Co}_{x} \mathrm{Ni}_{1-x}$ and hardly applies to $\mathrm{Co}_{x} \mathrm{Pd}_{1-x}$. Taking all the evidence together, roughly two cases can be distinguished when varying the concentration $x$ in a magnetic substitutional binary alloy, namely $(1) v\left(x, L_{0}\right)$ is proportional to $L_{0}(x)$ (LandauLifshitz behavior) provided that $\Delta A(x) /|\Delta A(x)|=\Delta K(x) /|\Delta K(x)|$, and (2) $v\left(x, L_{0}\right)$ seems to be proportional to $K(x)$, whenever $\Delta A(x) /|\Delta A(x)|=-\Delta K(x) /|\Delta K(x)|$, where $\Delta A(x)=d A(x) / d x$ and $\Delta K(x)=d K(x) / d x$.

In Ref. [7] for $1500 \mathrm{~nm}$ wide, $20 \mathrm{~nm}$ thick permalloy wires of length $80 \mu \mathrm{m}$ a velocity of about $40-60 \mathrm{~cm} / \mathrm{s}$ is reported for current densities $j>1.05 \times 10^{12} \mathrm{~A} / \mathrm{m}^{2}$, while for $j$ less than about $0.9 \times 10^{12} \mathrm{~A} / \mathrm{m}^{2}$ the velocity is about 10 $\mathrm{cm} / \mathrm{s}$. Considering that in the present calculations only the classical LLG equation (no spin torque term) is used and that a two-dimensional translational invariant system serves as a model for nanowires, in particular for low current densities, the values listed Table II, namely about $1 \mathrm{~cm} / \mathrm{s}$, for $\alpha=1$, agree reasonably well with their experimental counterparts. This is true even if in Eq. (25) a value of $\alpha$ of 0.1 or even less would be used, since $v\left(x, L_{0}\right)$ scales directly with $\alpha$. Therefore, it seems that despite all assumption made (such as a domain profile that does not change during motion) in terms of the presented approach material-specific velocities can be evaluated based entirely on parameters 

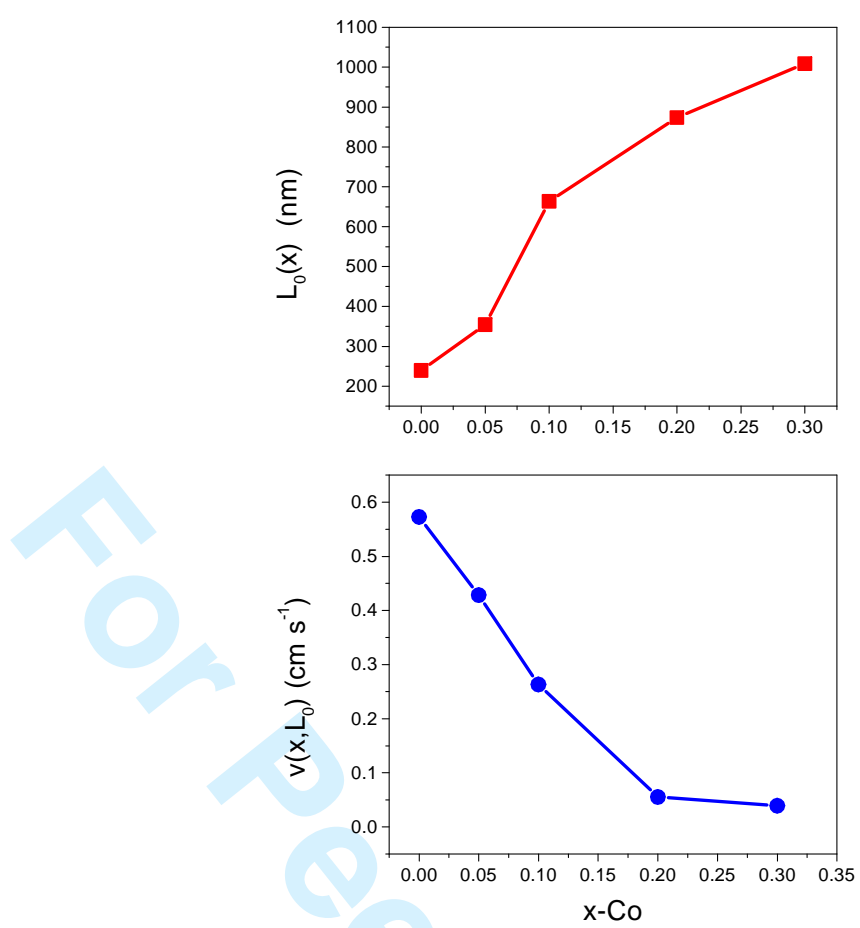

FIG. 4: (colour online) $\mathrm{Co}_{x} \mathrm{Ni}_{1-x}$.Equilibrium width $L_{0}(x)$ and velocity $v\left(x, L_{0}\right)$ for $0 \leq x \leq 0.3$.

obtained via an ab-initio fully relativistic approach. Finally, it should be noted that it would be extremely difficult to find "reasonable" parameters to predict concentration-dependent domain wall motions using micormagnetic schemes.

This work has been supported by the Deutsche Forschungsgemeinschaft in the framework of the project B3 of the SFB 668 and the Cluster of Excellence "Nanospintronics".

[1] L. Thomas, M. Hayashi, X. Jiang, R. Moriya, C. Rettner and S. S. P. Parkin, Nature 443, 197 (2006).

[2] M. Hayashi, L. Thomas, C. Rettner, R. Moriya, X. Jiang, and S. S. P. Parkin, Phys. Rev. Lett. 97, 207205 (2006).

[3] M. Hayashi, L. Thomas, C. Rettner, R. Moriya, and S. S. P. Parkin, Nature Phys. 3, 21 (2007).

[4] M. Hayashi, L. Thomas, C. Rettner, R. Moriya, Y. B. Bazaliy, and S. S. P. Parkin, Phys. Rev. Lett. 98, 037204 (2007).

[5] Y. Nakatani, A. Thiaville, and J. Miltat, Nature Mat. 2, 521 (2003).

[6] P. J. Metaxas, J. P. Jamet, A. Mougin, M. Cormier, J. Ferré, V. Baltz, B. Rodmacq, B. Dieny, and R. L. Stamps, Phys. Rev. Lett. 99, 217208 (2007).

[7] T. A. Moore, M. Kläui, L. Heyne, P. Möhrke, D. Backes, J. Rhensius, U. Rüdiger, L. J. Heyderman, J.-U. Thiele, G. Woltersdorf, C. H. Back, A. Fraile Rodríguez, F. Nolting, T. O. Mentes, M. Á. Niño, A. Locatelli, A. Potenza, H. Marchetto, S. Cavill, and S. S. Dhesi, Phys. Rev. B 80, 132403 (2009).

[8] A. Mougin and M. Cormier and J. P. Adam and P. J. Metaxas and J. Ferré, Europhys. Lett. 78, 57007 (2007).

[9] A. Thiaville and Y. Nakatani and J. Miltat and Y. Suzuki, Europhys. Lett. 69, 990 (2005).

[10] V. P. Antropov, M. I. Katnelson, M. van Schilfgaarde, and B. Harmon, Phys. Rev. Lett. 75, 729 (1995).

[11] G. M. Stocks, B. Újfalussy, B. Lazarovits, L. Szunyogh, M. Eisenbach, and P. Weinberger, Progr. Mat. Sci. 52, 371 (2007).

[12] D. Steiauf, J. Seib, and M. Fähnle, Phys. Rev. B 78, 020410 (2008).

[13] J. Schwitalla, B. L. Gyorffy, and L. Szunyogh, Phys. Rev. B 63, 104423 (2001).

[14] S. Gallego, P. Weinberger, L. Szunyogh, P. M. Levy, and C. Sommers, Phys. Rev. B 68, 054406 (2003).

[15] H. C. Herper and P. Entel, Phys. Rev. B 77, 174406 (2008).

[16] A. J.Freeman and K.Nakamura, J. Magn. Magn. Mater. 321, 894 (2009).

[17] P. Weinberger, Phys. Rev. Lett. 98, 027205 (2007).

[18] P. Weinberger, Phys. Rev. Lett. 100, 017201 (2008).

[19] P. Weinberger, Phys. Rev. B 78, 172404 (2008).

[20] P. Weinberger, Phil.Mag. 89, 1933 (2009).

[21] L. D. Landau \& J. M. Lifshitz, Phys. Z. SU 8, 153 (1935). 
[22] A. P. Malozemoff \& J. C. Slonczewski in Applied Solid State Science, Suppl.1 (Academic Press, New York, 1979).

[23] N. L. Schryer and L. R. Walker, J. Appl. Phys. 45, 5406 (1974).

[24] A. Vernes, B. L. Gyorffy, and P. Weinberger, Phys. Rev. B 76, 012408 (2007).

[25] P. Weinberger, Magnetic Anisotropies in Nanstructured Matter (CRC Boca Raton London New York, 2008).

[26] J. Zabloudil, R. Hammerling, L. Szunyogh and P. Weinberger, Electron Scattering in Solid Matter (Springer Berlin Heidelberg New York, 2004).

[27] P. Weinberger, Phys. Rev. B 81, 104417 (2010).

[28] This can easily be seen considering the quantity $P(L) \vec{j}(L)$, the "spin-polarized current density", which is only quantum mechanically well-defined in the (non-relativistic) case of collinear magnetic structures. In principle for non-collinear magnetic structures this term has to be replaced by the so-called polarization density, introduced in Ref. [24].

[29] P. Weinberger, A. Vernes, B. L. Gyorffy, and L. Szunyogh, Phys. Rev. B 70, 094401 (2004); A. Vernes, P. Weinberger, and L. Szunyogh, Phys. Rev. B 72, 0112401 (2005). 
( $\mathrm{Ni}_{8} \mathrm{Fe}_{15}$. Top: Original (unshifted) layer-resolved band energies for $\mathrm{L}_{0}=1038 \mathrm{ML}$. The middle and the bottom entries refer to the band energies corresponding to the "left" and "right" edge of the original domain wall (full line) and the one shifted by $1 \mathrm{ML}$ (dashed line). $209 \times 297 \mathrm{~mm}(600 \times 600 \mathrm{DPI})$ 

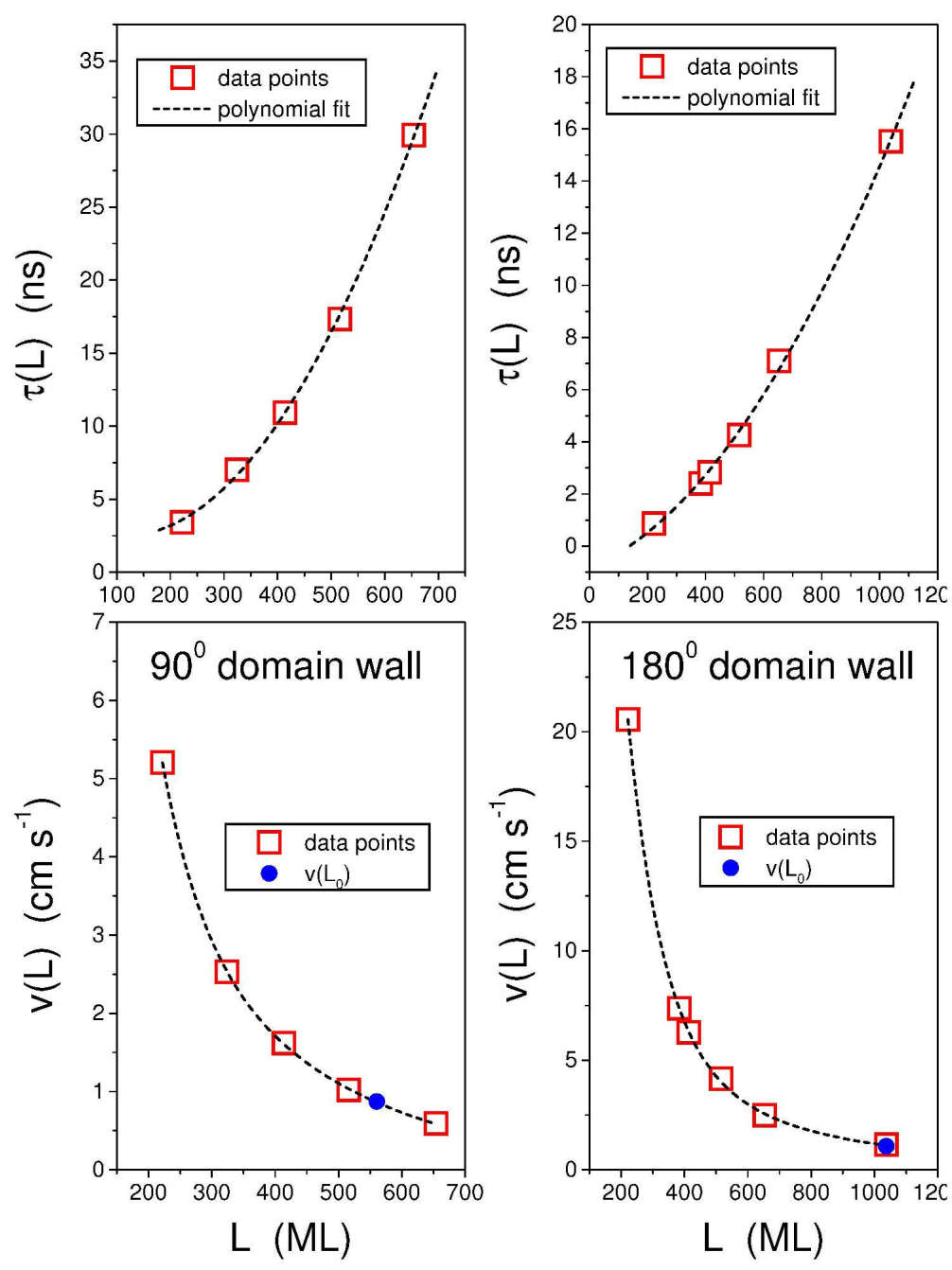

$\mathrm{Ni}_{8} \mathrm{Fe}_{15}$. Top row: fit of $\mathrm{T}(\mathrm{L})$ by a second order polynomial. Bottom row: domain wall velocities. Displayed are the cases of a $90^{\circ}$ (left column) and a $180^{\circ}$ (right column) domain wall. $209 \times 297 \mathrm{~mm}(600 \times 600 \mathrm{DPI})$ 

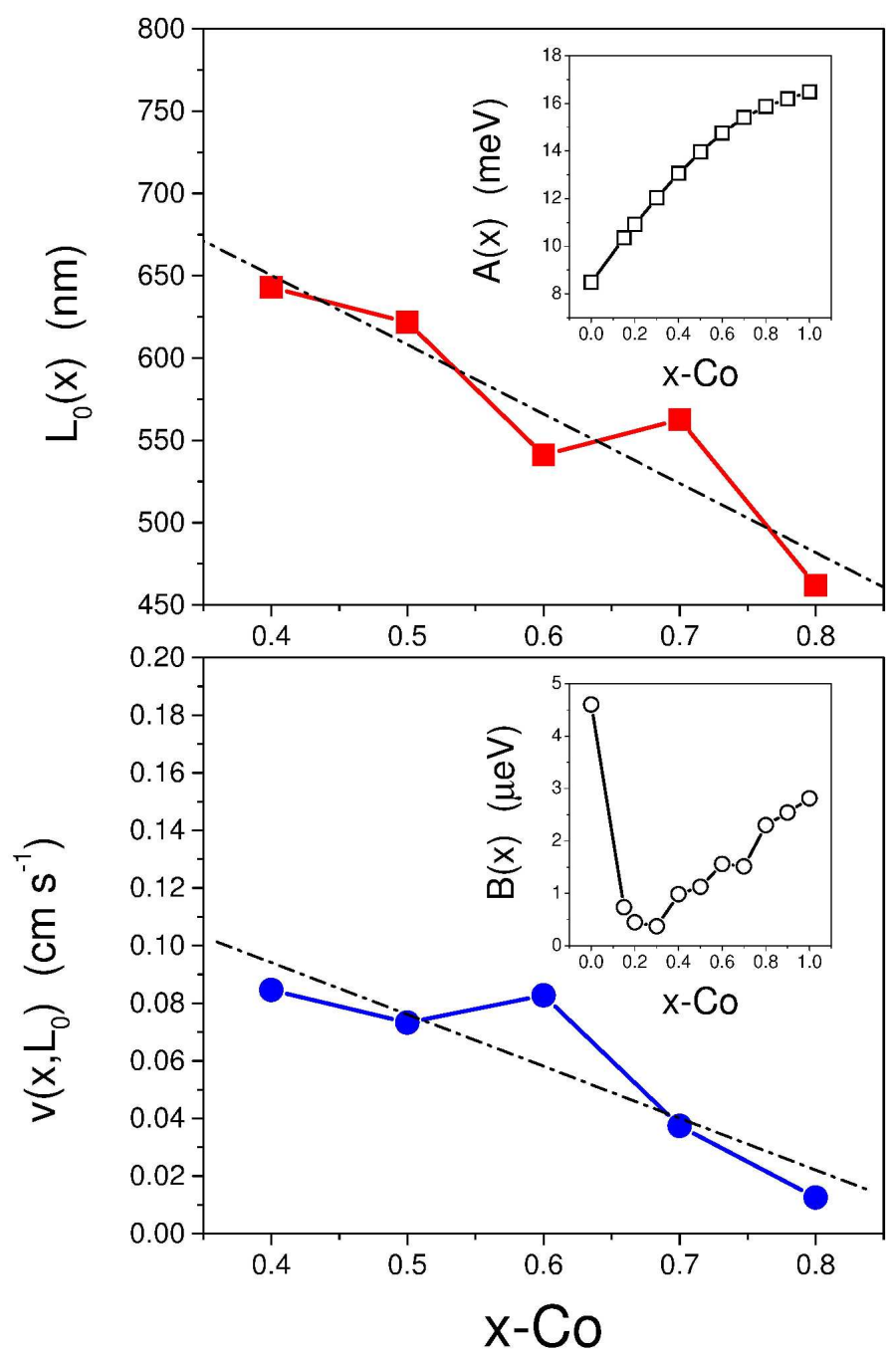

Co_ $\{x\} N i_{1}\{1-x\}$. Equilibrium width $L_{0}(x)$ (top) and velocity $v\left(x, L_{0}\right)$ as indicated in the range of $0.4<x_{-}\{\mathrm{Co}\} \leq 0.8$. The insets show the exchange (top) and anisotropy energy (top) data from Ref. $<$ cite $>$ dw-pw-3</cite $>$. $209 \times 297 \mathrm{~mm}(600 \times 600 \mathrm{DPI})$ 

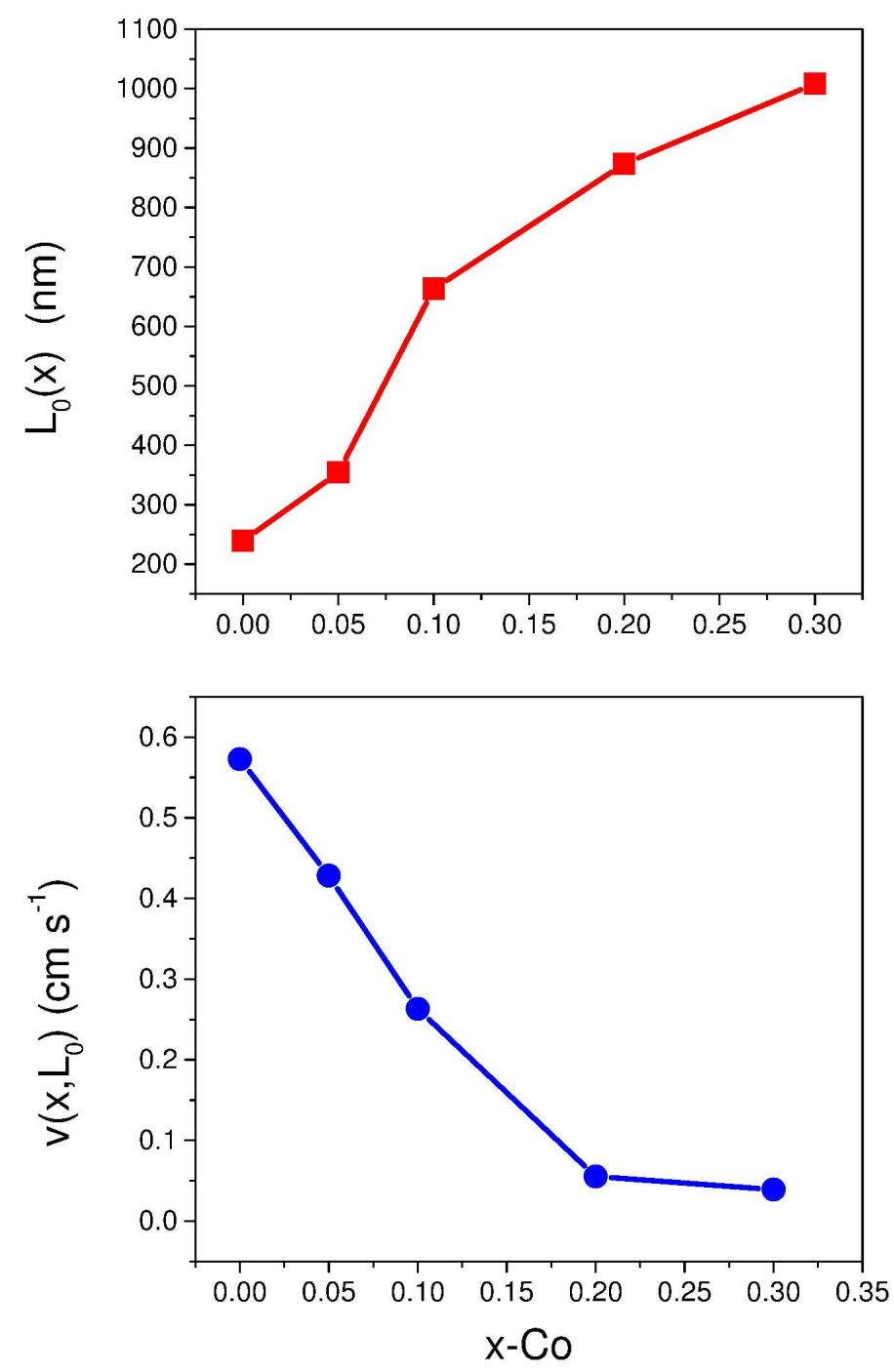

Co_ $\{x\} N N_{-}\{1-x\}$.Equilibrium width $L_{0}(x)$ and velocity $v\left(x, L_{0}\right)$ for $0 \leq x \leq 0.3$. $209 \times 297 \mathrm{~mm}(600 \times 600 \mathrm{DPI})$ 

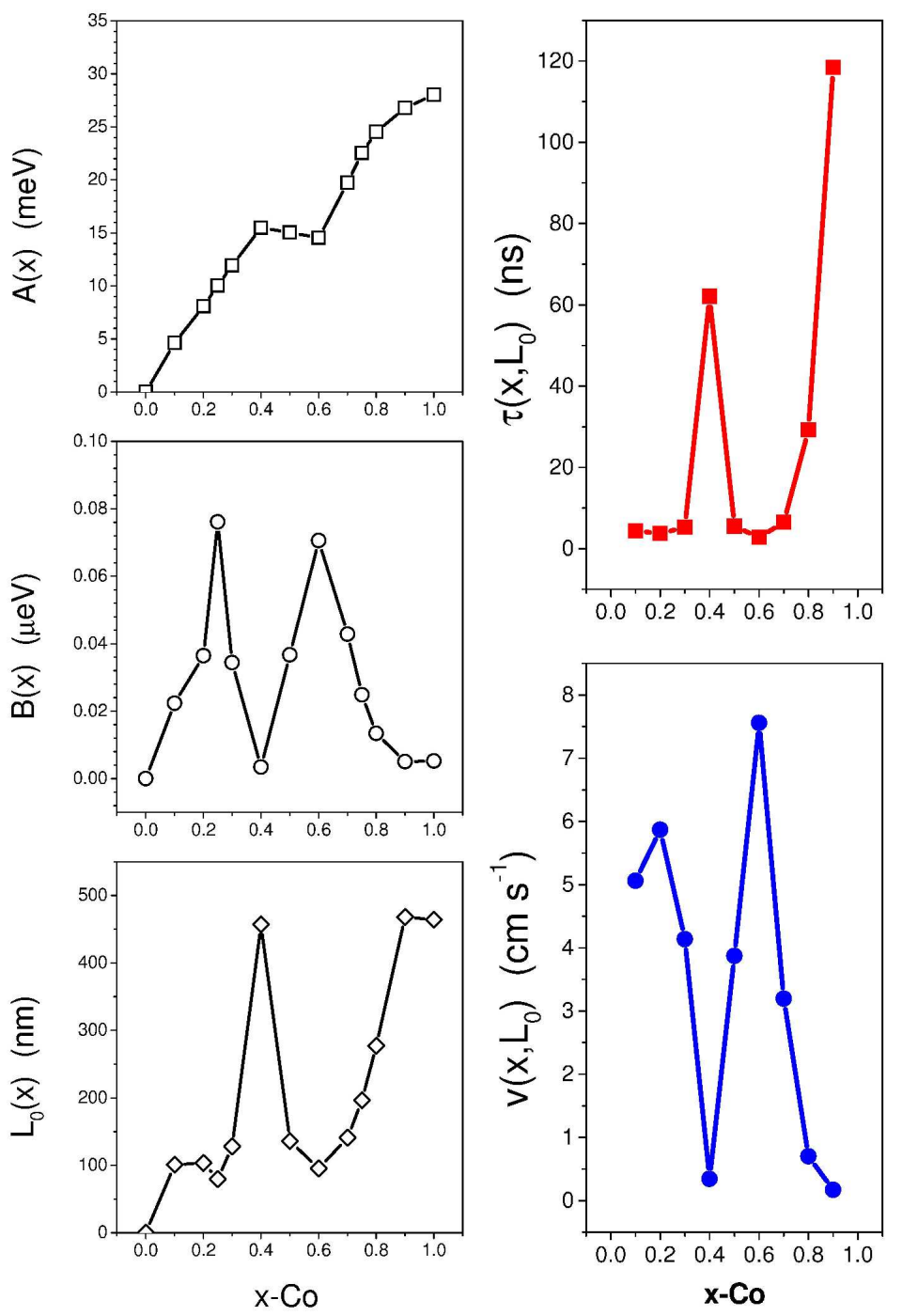

Co_ $\{x\} P d \_\{1-x\}$.Shift time $T\left(x, L_{0}\right)$ width (top, right) and velocity $v\left(x, L_{0}\right)$ (bottom, right) versus the Co concentration. For comparison in the left colum the data for $A(x), B(x)$ and $L_{0}(x)$ from Ref. $<$ cite $>$ dw-pw- $4</$ cite $>$ are displayed. $209 \times 297 \mathrm{~mm}(600 \times 600 \mathrm{DPI})$ 

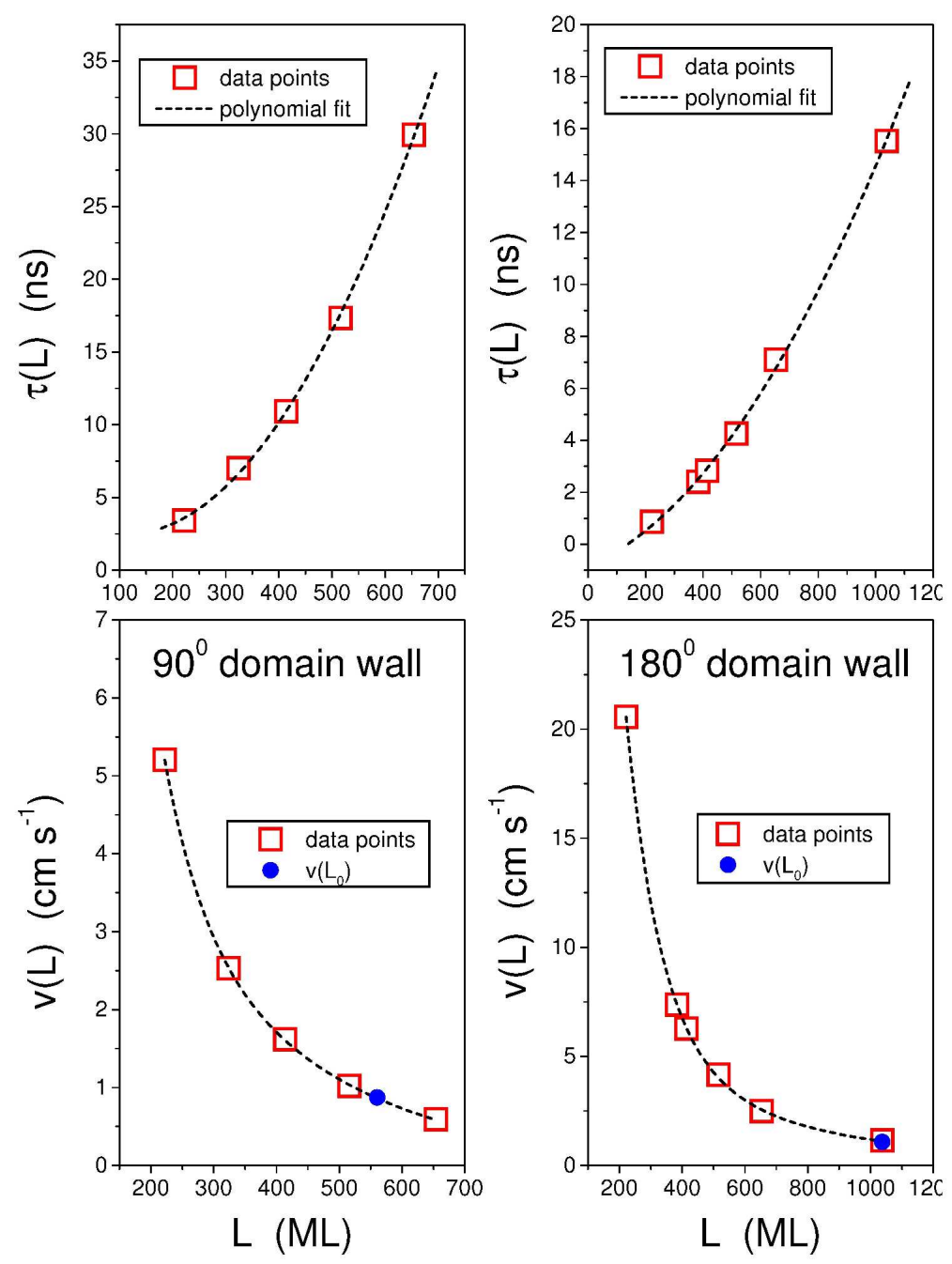

$\mathrm{Ni}_{8} \mathrm{Fe}_{15}$. Top row: fit of $\mathrm{T}(\mathrm{L})$ by a second order polynomial. Bottom row: domain wall velocities. Displayed are the cases of a $90^{\circ}$ (left column) and a $180^{\circ}$ (right column) domain wall. $209 \times 297 \mathrm{~mm}(600 \times 600 \mathrm{DPI})$ 

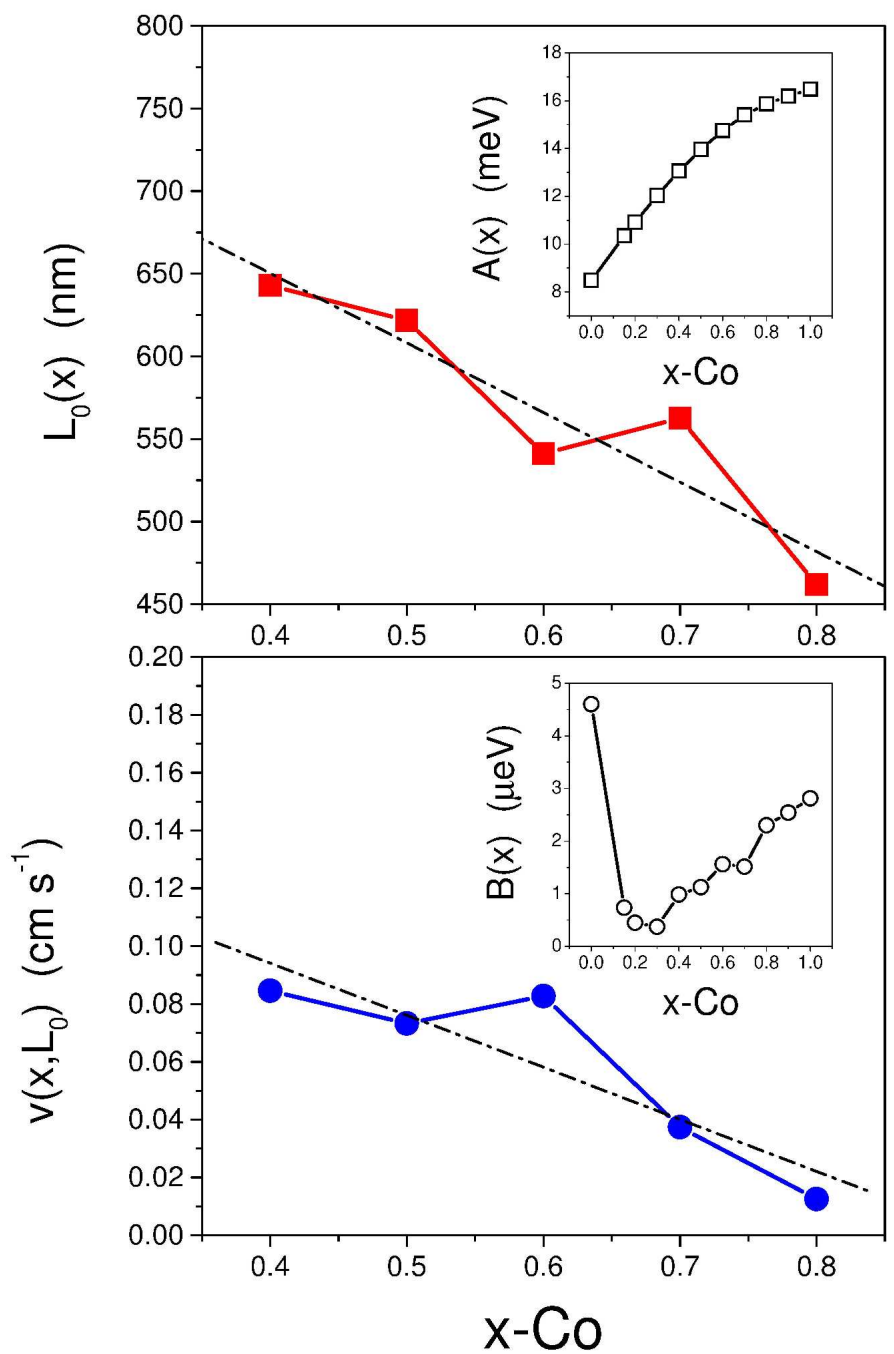

Co_ $\{x\} N i \_\{1-x\}$. Equilibrium width $L_{0}(x)$ (top) and velocity $v\left(x, L_{0}\right)$ as indicated in the range of $0.4<x_{-}\left\{\mathrm{Co}_{0} \leq 0.8\right.$. The insets show the exchange (top) and anisotropy energy (top) data from Ref. $<$ cite $>$ dw-pw-3</cite $>$. $209 \times 297 \mathrm{~mm}(600 \times 600 \mathrm{DPI})$ 

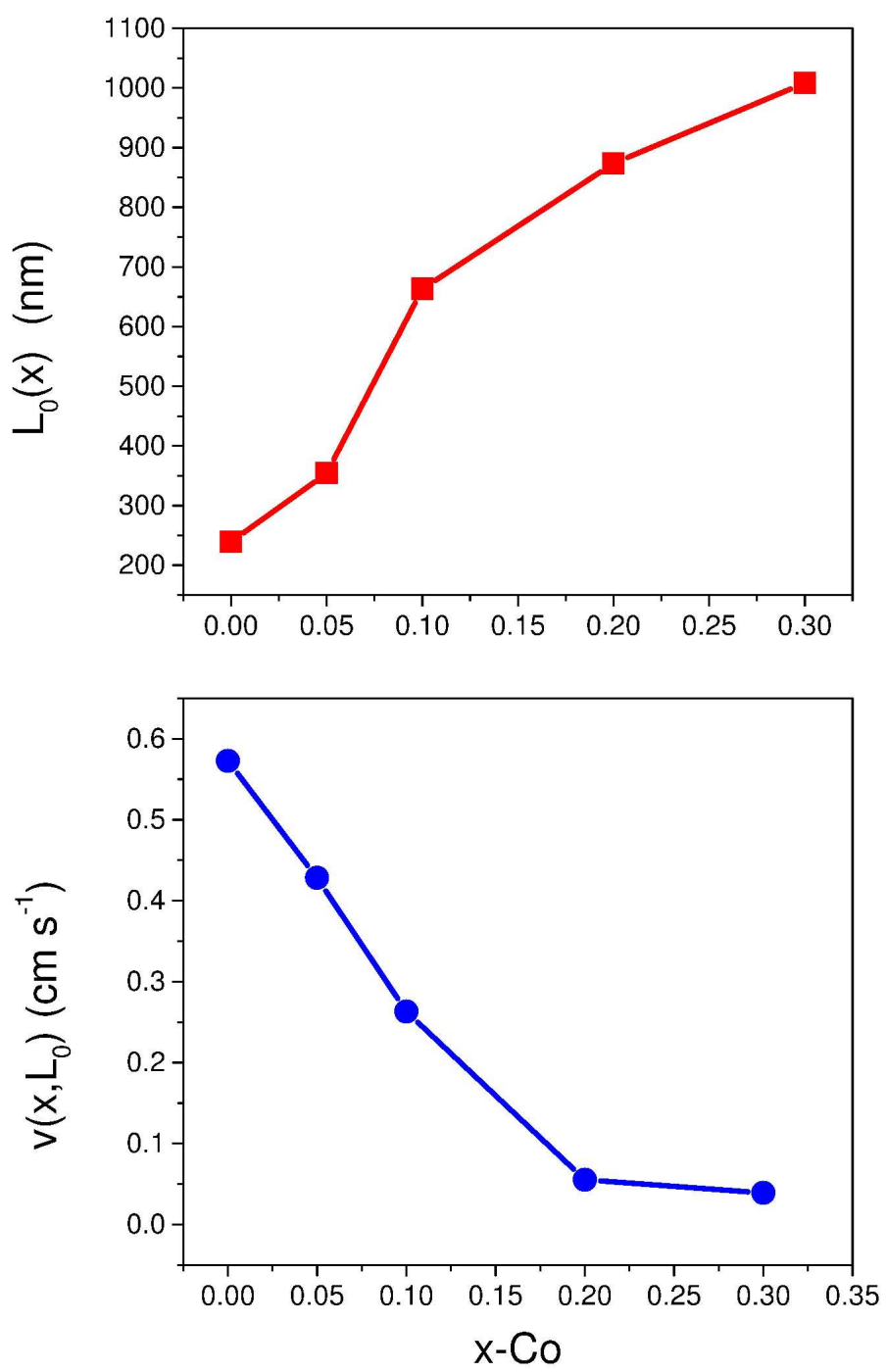

Co_ $\{x\} N i \_\{1-x\}$. Equilibrium width $L_{0}(x)$ and velocity $v\left(x, L_{0}\right)$ for $0 \leq x \leq 0.3$. $209 \times 297 \mathrm{~mm}(600 \times 600 \mathrm{DPI})$ 

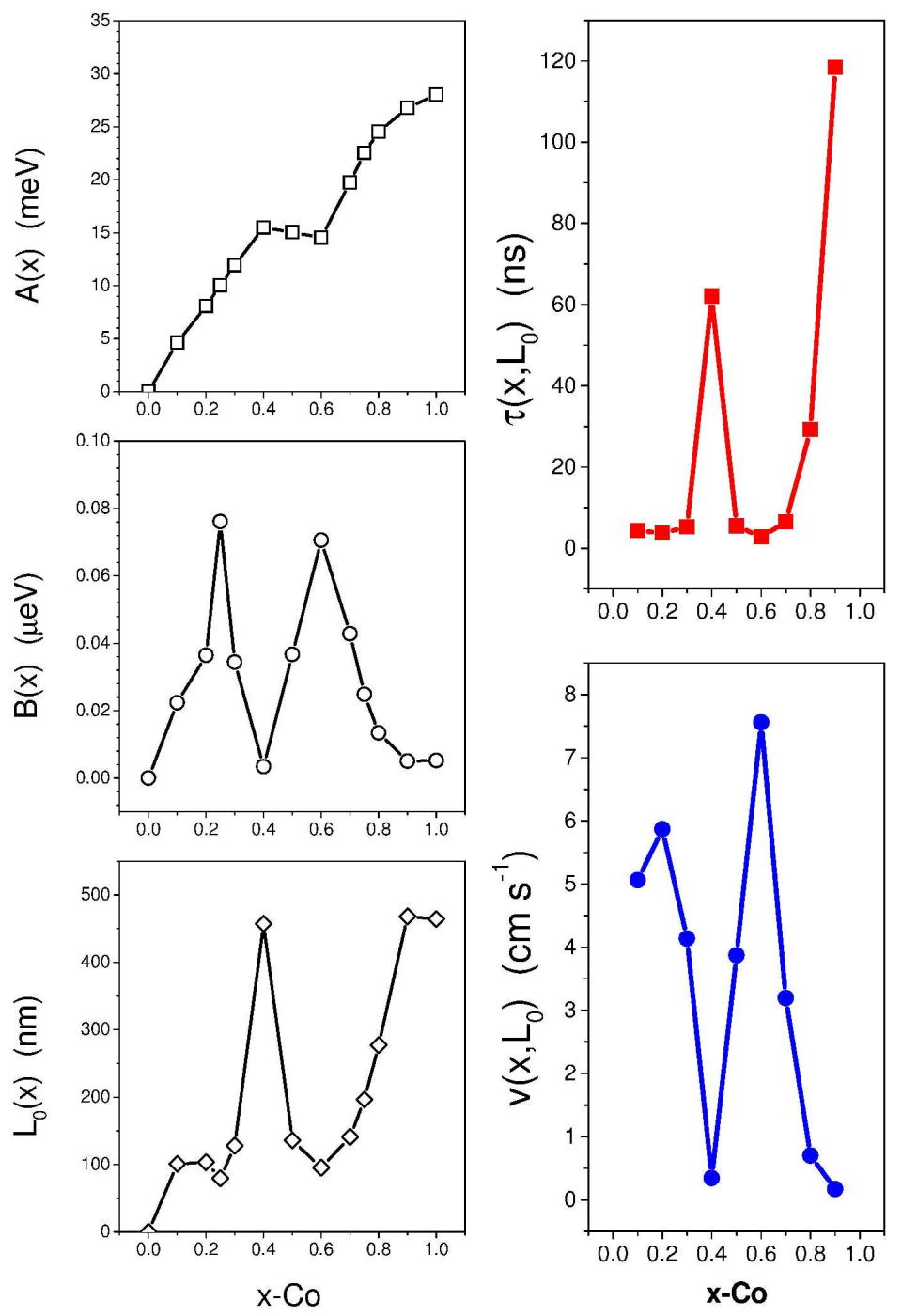

Co_ $\{x\} P d \_\{1-x\}$.Shift time $T\left(x, L_{0}\right)$ width (top, right) and velocity $v\left(x, L_{0}\right)$ (bottom, right) versus the Co concentration. For comparison in the left colum the data for $A(x), B(x)$ and $L_{0}(x)$ from Ref. $<$ cite $>$ dw-pw-4</cite $>$ are displayed. $209 \times 297 \mathrm{~mm}(600 \times 600 \mathrm{DPI})$ 